\title{
5G Low-latency communication in Virtual Reality services: Performance requirements and promising solutions
}

\author{
DRAGORAD MILOVANOVIC, ZORAN BOJKOVIC \\ University of Belgrade \\ REPUBLIC of SERBIA \\ MADHAVSINGH INDOONUNDON, TULSI PAWAN FOWDUR \\ Department of Electrical and Electronic Engineering \\ University of Mauritius \\ REPUBLIC of MAURITIUS
}

\begin{abstract}
This work explores low-latency communication enabled by the Fifth Generation of mobile networks $(5 \mathrm{G})$ and its integration in emerging virtual reality (VR) applications. Mobile VR is at the crossroad between mobile broadband (eMBB) and ultra-reliable and low-latency communication (uRLLC) services. The current trends in wireless multimedia communication are introduced. A review of mobile VR and haptics requirements is made and end-to-end (E2E) latency components in uRLLC downlink physical layer are described. Moreover, non-orthogonal multiple access (NOMA) in short-packet downlink transmission is discussed. It is pointed out that NOMA is a promising technology for realizing massive mobile connectivity and more comprehensive research is required to better understand its suitability for VR applications.
\end{abstract}

Key-Words: 5G, eMBB, uRLLC, NOMA, VR services

Received: January 23, 2021. Revised: June 9, 2021. Accepted: June 30, 2021. Published: July 9, 2021.

\section{Introduction}

The fifth generation of mobile communication networks, $5 \mathrm{G}$, is the generation that will replace the current $4 \mathrm{G}$ mobile wireless networks. $5 \mathrm{G}$ introduces a new network architecture and advanced technologies that can support different use cases. 5G networks constitute a more complex ecosystem than $4 \mathrm{G}$ and have more heterogeneity and more dynamicity $[1,2,3,4]$. Mobile video traffic is found to be increasing exponentially and by 2021 , it is expected to take up to $70 \%$ to $80 \%$ of all data traffic. There are increasing demands for the support of high video resolutions of up to $4 \mathrm{~K}$, for $360^{\circ}$ video and VR services, and for mobile augmented reality (AR) applications. 5G wireless networks provide the necessary advanced technologies which can support data rates in the order of Gbps, massive connection density, and reliable connectivity which will be the pillars for a panoply of deployment scenarios. The three classes of innovative $5 \mathrm{G}$ services are as follows.

- eMBB (enhanced Mobile Broadband) services: These services require a network capable of handling very high data rate applications and very high user density. Such services include highquality VR and AR applications which are expected to bring a drastic increase in video consumption in networks.

- mMTC (massive Machine Type Communications) services: These services require a network capable of supporting a large number of devices that typically communicate using small volumes of data. Devices supporting mMTC services are required to be cost and energy-friendly.

- uRLLC (ultra-Reliable and Low-Latency Communications) services: These services have stringent latency and reliability requirements. uRLLC services have a low packet error rate requirement in the order of 10-5 and a low E2E transmission latency requirement of under $1 \mathrm{~ms}$. These requirements are crucial for the future development of smart networks $[5,6]$.

Mobile VR is at the crossroad between eMBB and uRLLC services. Despite the multiple efforts which have been made in 5G technology lately, there remain multiple challenges in the field of multimedia transmission. Artificial intelligence (AI) network technologies that may help $5 \mathrm{G}$ to overcome various challenges are in development. These technologies may enhance the integration of $5 \mathrm{G}$ in vertical industries and will allow $5 \mathrm{G}$ to meet diverse market requirements. 
In this paper, the use of 5G to provide low-latency services is studied. Both VR and haptics requirements are reviewed. The rest of this paper is organized as follows. A short overview of latency requirements is given followed by the description of emerging services in 5G. Then, the adoption of NOMA into short-packet downlink transmission is analyzed. Afterward, the challenges and future trends for uRLLC physical layer security are listed. Finally, concluding remarks and references are provided

\section{5G multimedia communication}

Today $5 \mathrm{G}$ is regarded as the key network technology being developed to satisfy challenging network requirements. It will be the enabler of a hyperconnected society consisting of a broad amount of connected users who are capable of the rapid exchange of large volumes of data. These users will also be able to utilize the network for using and even providing services at a higher level of quality of experience (QoE). 3D immersive media is evolving to support new types of applications and services. The evolution includes the provision of higher resolution, new types of immersive perception (use of augmented/virtual/mixed reality), and new types of devices. This evolution of immersive technology has the aim of coping with limitations in terms of interaction, perception of distances, coexistence, and presence. Furthermore, the use of $\mathrm{AI}$ and machine learning (ML) can provide personalized immersive media and analytics in different environments to endusers.

\subsection{Mobile Virtual Reality}

VR is a use case in which uRLLC is vital since the human eye needs to be able to track movement accurately and smoothly at low latencies of around $20 \mathrm{~ms}$ to avoid motion sickness. The interest in wireless VR has been existent since 2016. Wireless VR requires user data to be delivered at a rate of up to $5 \mathrm{Gbps}$ at low latency. Interconnected VR services require resources to be efficiently distributed, high QoE levels to be guaranteed, and steady interconnections between users to be maintained. Therefore this presents new challenges in videoaudio encoding, transmission, manipulation, synchronization, and storage [7]. Wireless VR provides a shared haptic environment in which multiple users are allowed to interact via physically coupled VR simulations. This unlocks the possibility to support applications such as micro-assembly and telesurgery which also require very high levels of sensitivity and accuracy for the manipulation of objects [8].

\subsection{Haptics requirements}

Haptics refers to any form of interaction that involves touch. It consists of both tactile and kinesthetic sensing. Haptics deals with information acquisition and objects manipulation through touch. It also comprises of manual exploration, manipulation by humans and machines, and man-to-machine interactions in the physical, virtual, teleoperated, or networked environment [9].

The main concern raised by the increasing research attention in haptic interfaces for VR, AR, human-machine interactions, and human-robot interaction is the transmission of error-free information through touch. Psychophysical techniques have widely been applied to the development of both hardware and software in the field of haptic research over the past decades.

\subsection{Latency requirements}

To properly implement a network that supports wireless VR, the low-latency requirement for both visual and haptic information must be taken into account. Some components of the VR environment require E2E latencies lower than 15 to $20 \mathrm{~ms}$. VR systems comprise of various system components which significantly increase the joint computing and communication latency budget. One major component of the communication latency budget is the E2E wireless latency which encompasses sensor sampling latency, image processing latency, frame computing latency, network latency, and display refresh latency. Latency in the sensor display is imperceptible, whereas display latency must be dropped from $10 \mathrm{~ms}$ to $5 \mathrm{~ms}[10,11,12]$.

E2E latency also includes over-the-air delay and delay due to retransmissions when required. A breakdown of the latency in the physical layer is given as follows:

$$
\mathrm{T}_{\mathrm{L}}=\mathrm{T}_{\text {ttt }}+\mathrm{T}_{\text {prop }}+\mathrm{T}_{\text {proc }}+\mathrm{T}_{\text {ret }} \mathrm{X}+\mathrm{T}_{\text {sig }}
$$

$\mathrm{T}_{\text {ttt }}$ time-to-transmit delay

$\mathrm{T}_{\text {prop }}$ propagation time of the signal from the transmitter to the receiver

$\mathrm{T}_{\text {proc }}$ encoding and decoding time including channel estimation time during the initial transmission

$\mathrm{T}_{\text {retx }}$ delay added due to retransmission

$\mathrm{T}_{\text {sig }}$ time of pre-processing signaling messages exchange such as scheduling grant, channel training, and feedback, queuing delay and feedback, and connection request.

An illustration of the uRLLC downlink physical latency breakdown is given in Fig. 1. The latency breakdown consists of both random and deterministic elements which can be either fixed or scale. The minimum latency of the system is determined by the deterministic elements. On the other hand, the 
random elements add additional latency in the system and more specifically at its tails. Deterministic latency elements consist of the time to transmit both information and overhead and of the waiting times between transmission. Random latency elements include the retransmission time of information and overhead when required, delays added due to queuing, random backoff times, and other processing delays.

\subsection{Emerging 5G low-latency services}

In recent years, the need for low-latency communication has become crucial, especially in applications such as manufacturing, transportation, robotics, health-care, VR/AR, and education. Always-on hyper-connected devices that require uRLLC include smart wearable devices, smart home appliances, sensors, autonomous cars, cognitive mobile devices, robots, and drones. Some of the applications even have additional stringent network capabilities such as high reliability and enhanced security. Factory automation requires real-time manipulation of machines to offer quick production lines and limit human interventions. Factory automation has an E2E latency requirement of between $0.25 \mathrm{~ms}$ to $10 \mathrm{~ms}$ and a packet loss rate requirement below $10^{-9}$. In an intelligent transportation system (ITS), the latency requirement needs to be tailored for each case. In autonomous vehicles, the maximum E2E latency which can be tolerated for each transmission is $10 \mathrm{~ms}$. For efficiency services control, the latency is required to be in the range of $10 \mathrm{~ms}$ to $100 \mathrm{~ms}$ and the packet loss rate must be in the range of $10^{-3}$ to $10^{-5}$. Real-time remote control with synchronous visual and haptic feedback is crucial for the use of robots and telepresence applications. In such scenarios, the system response time combined with network delays should be below a few milliseconds. $5 \mathrm{G}$ will provide the network infrastructure which will cater for the support of large capacity, high reliability, high availability, and seamless mobility requirements.

With the latency reduced to only a few milliseconds, AR technology will allow visual and haptic information to be interacted with remotely in real-time and will be the enabler of innovative systems and applications including driver-assistance systems, remote maintenance systems, enhanced navigation guides, telemedicine (telediagnosis, telesurgery, telerehabilitation), remote education, and improved emergency services. It should thus be noted that minor network failures that may drastically increase the latency will negatively impact missioncritical services running on the $5 \mathrm{G}$ network. In the education sector, low latency tactile internet with roundtrip delays of $5 \mathrm{~ms}$ to $10 \mathrm{~ms}$ will ease the sharing of visual, auditory, and tactile information between teachers and students.

\section{Low-latency and secure communication}

The 5G network's requirement to support high user density poses challenges to the current orthogonal multiple access technologies. Non-orthogonal multiple access (NOMA) offers the possibility to connect more than one user equipment to the network, using the same time, frequency, space, and code resources $[13,14,15]$. Therefore, NOMA is a good candidate which can meet can both eMBB and uRLLC requirements. There exist different types of NOMA techniques including power-domain and code-domain NOMA. To implement power-domain NOMA, the transmitter is designed to incorporate a superposition coding module and the receiver is designed to incorporate a successive interference cancellation (SIC) module. Receivers with SIC modules, which receive combined signals from multiple sources, first decode the strongest signal. Then they subtract the decoded strongest signal from the received combined signals and proceed with decoding the second strongest signal. The process is repeated until all signals are individually recovered. Imperfect SIC may degrade the performance of NOMA by introducing residual interference [16].

\subsection{Short-packet downlink transmission}

With flexible user scheduling, scheduling delays are minimized as multiple users are served in a timely manner and can therefore be adopted in 5G's uRLLC service class. There are multiple advantages of applying NOMA to the transmission of short-packets at low-latency [17]. First of all, NOMA provides a high spectrum efficiency which compensates for the performance degradation caused by short-packet communications (Fig. 2) and thus enhances network connectivity. NOMA also enables efficient resource utilization because access to the network is provided to multiple users via the same non-orthogonal communications resources. Furthermore, in the downlink, NOMA allows users with poor channel conditions to specifically receive signals with increased power levels. In the uplink, strong signals from users in good channel conditions are subtracted from the signal originating from a peer user with poor channel conditions to perform accurate demodulation. Hence user fairness is achieved in both uplink and downlink.

The use of NOMA is beneficial in short-packet communications. Both channel gains and packet length should be considered in the user pairing 
strategy for NOMA. The packet length may vary depending on the different delay constraints of the system. SIC is performed by detecting the whole packet and so, the packet in each signal should be integrally recovered for the receiver to be able to accurately subtract them from the received combined signal. In practice, SIC is not perfect and may lead to both uplink and downlink severe co-channel interference when using NOMA [18].

\section{2 uRLLC Physical layer security}

The key requirements of uRLLC are high reliability and low latency. Also, VR applications require highsecurity infrastructures. The physical layer security (PLS) techniques are based on the inherent randomness of the wireless channel. Thus, physical layer security has low complexity and thus introduces negligible latency. Research on physical layer security is still immature and has low attention. Hence the implementation of PLS in practical uRLLC scenarios introduces challenges. One such challenge is the transmission of accurate channel state information (CSI) at ultra-low latency, ultrahigh reliability, and moderate levels of security [19, 20, 21, 22].

PLS techniques should be able of securing wireless communication without introducing additional delay and without using up communication resources significantly. The distribution and management of secret keys and the addition of extra security mechanisms on other layers must be avoided to reduce signaling overhead. PLS techniques for short-packet communication are of practical interest in VR applications.

\section{Concluding remarks}

This article explored 5G low-latency communication and its integration with emerging technologies to support VR services. The current trends of wireless multimedia communication including mobile VR and haptics are described. Latency requirements and sources of E2E in the physical layer are also introduced.

The 5G low-latency service category accommodates emerging use cases with strict reliability requirements. Low latency and reliable communication call for new solutions and fundamental research cutting across several disciplines. VR is an application designed to immerse users in a virtual environment incorporating visual, auditory, and haptic elements for emulating the real world and has strict network requirements. Finally, NOMA combined with SIC technology is reviewed and proposed as a future research direction.
References:

[1]P.Popovski et al., A perspective on time towards wireless 6G, arXiv:2106.04314, 2021, pp.1-24

[2]D.Milovanovic al. Advanced human centric 5GIoT in a Smart City: Requirements and challenges, in Proc. Human-Centered Computing (HCC'19) vol. 11956, Springer 2019, pp.285-296

[3]Z.Bojkovic, D.Milovanovic, T.P.Fowdur (Eds), 5G Multimedia communications: technologies, multiservices, deployment, CRC Press, 2020.

[4]D.Milovanovic, Z.Bojkovic, Optimal deployment of $5 G$ network slicing based on complex network theory, in Proc. TINKOS 2019, pp.1-2

[5]M.S.Elbamby, C.Perfecto, K.Doppler, Toward low-latency and ultra-reliable virtual reality. IEEE Network, vol.32, no.3, 2018, pp.78-84

[6]B.Ali et al., 5G technologies for Smart Railways, Proc. of the IEEE, vol.108, no.6, 2001, pp.856893

[7]D.Milovanovic, D.Kukolj, Emerging levels of immersive experience in MPEG-I video coding, IEEE COMSOC MMTC Communications Frontiers, Vol.13, No.1, January 2018, pp. 24-26

[8]H.Z.Tan, The technical committee on haptic. IEEE Robotics \& Automation Magazine, vol.15, no.1, 2008, pp.16

[9]H.Z.Tan et al., Methodology for maximizing information transmission of haptic devices: A survey. Proc. of the IEEE, vol.108, no.6, 2020, pp.945-965

[10] P. Popovski et al., Wireless access for ultrareliable low-latency communication: Principles and building blocks, IEEE Network, vol.32, no.2, 2018, pp.16-23

[11] M.Bennis, M.Debbah, H.V.Poor, Ultrareliable and low-latency wireless communication: Tail, risk, and scale, Proc. of the IEEE, vol.106, no.10, pp.1834-1853, Sep. 2018.

[12] I. Parvez, A. Rahmati, I. Guvenc, A. I. Sarwat, $\mathrm{H}$. Dai, A survey on low latency towards $5 \mathrm{G}$ : RAN, core network and caching solutions, IEEE Communications Surveys \& Tutorials, vol. 20, no. 4, 2018, pp. 3098-3130

[13] T.Xiang et al., Physical Layer security in Cognitive Radio inspired NOMA network. IEEE Journal of Selected Topics in Signal Processing, Vol.13, No.3, 2019, pp.700-714

[14] Z.Ding et al., Application of Non-Orthogonal Multiple Access in LTE and 5G networks. IEEE Communications Magazine, Vol.55, No.2, 2017, pp.185-191

[15] Y.Lieng et al. Non-orthogonal random access for $5 \mathrm{G}$ networks. IEEE Wireless Communications, Vol.16, No.7, 2017, pp.48174831 
[16] D.Wan et al., A promising NOMA based networking architecture: Motivation, conception and education. IEEE Wireless Communications, Vol.26, No.5, 2019, pp.152159

[17] X.Sun et al., Short-packet downlink transmission with NOMA. IEEE Trans. Communications, Vol.17, No.7, 2019, pp.45504554

[18] Z.Xiang et al., NOMA-assisted secure shortpacket communications in IoT. IEEE Wireless Communications, Vol.27, No.4, 2020, pp.8-15

[19] H.Ji et al., Ultra-Reliable and Low-Latency Communications in 5G Downlink: Physical
Layer aspects. IEEE Wireless Communications, Vol.25, No.3, 2018, pp124-130

[20] R.Chen et al., Physical Layer security for UltraReliable and Low-Latency communication. IEEE Wireless Communications, Vol.26, No.5, 2019, pp.6-11

[21] Y.Alfadhli, Y.-W.Chen, S.Liu, S.Shen, S.Yao, D. Guidotti, S. Mitani, G.-K. Chang, Latency performance analysis of low layers function split for URLLC applications in 5G networks, Computer Networks, vol.162, 2019, pp.1-18

[22] D.Milovanovic, Z.Bojkovic, 5G Mobile networks: What is next? IARAS International Journal of Communications, Vol.4, 2019, pp.1-9

\section{Creative Commons Attribution License 4.0 (Attribution 4.0 International, CC BY 4.0)}

This article is published under the terms of the Creative Commons Attribution License 4.0

https://creativecommons.org/licenses/by/4.0/deed.en_US
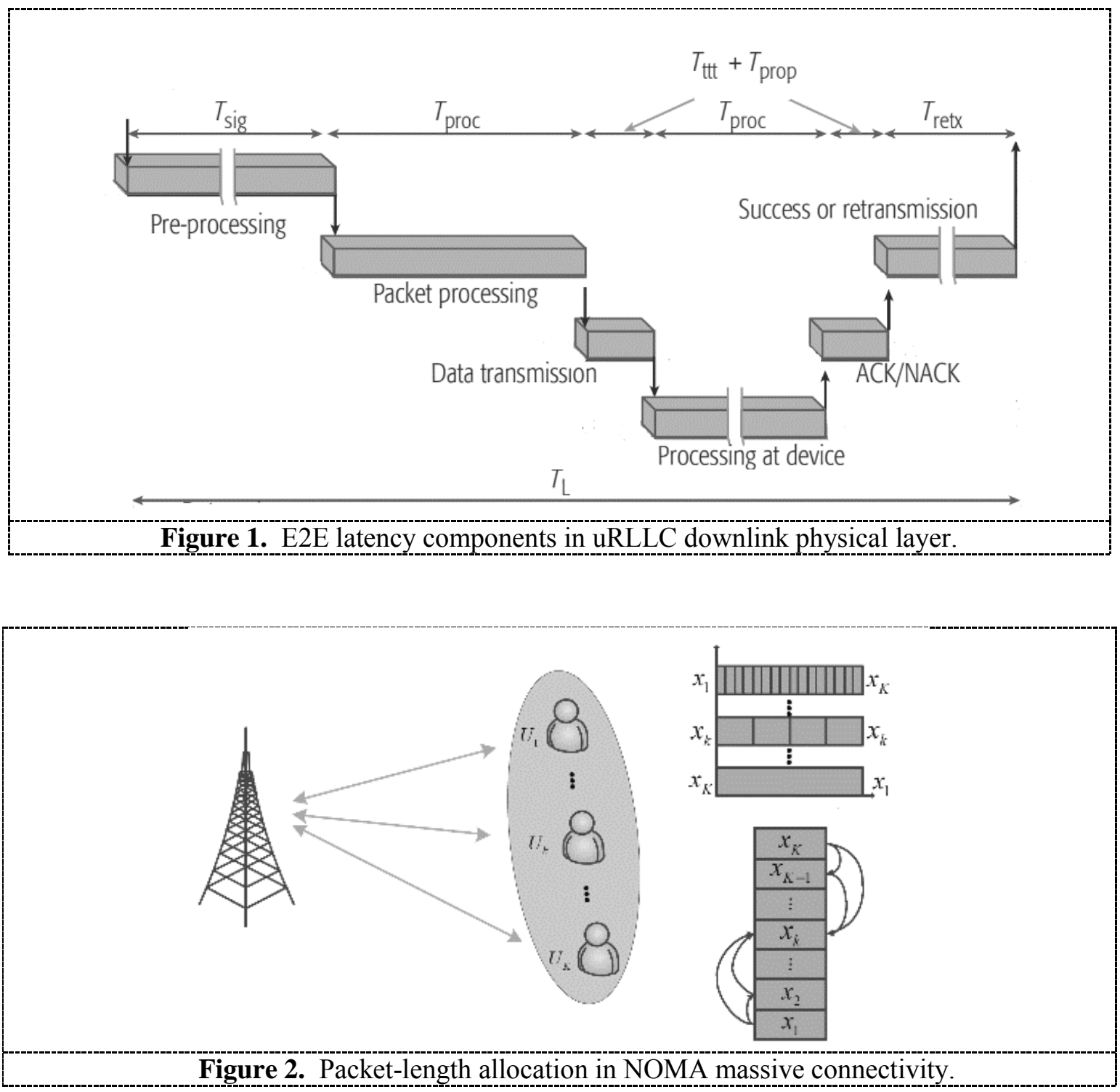